\title{
Iterative Learning Control as a Framework for Human-Inspired Control with Bio-Mimetic Actuators
}

\author{
Franco Angelini ${ }^{1,2}[0000-0003-2559-9569]$, Matteo Bianchi ${ }^{1[0000-0003-4747-1697]}$, \\ Manolo Garabini ${ }^{10000-0002-5873-3173]}$, Antonio Bicchi ${ }^{1,2}[0000-0001-8635-5571]$, and \\ Cosimo Della Santina ${ }^{3,4,5}[0000-0003-1067-1134]$ \\ 1 Centro di Ricerca "Enrico Piaggio" and DII, Università di Pisa, Italy \\ ${ }^{2}$ Soft Robotics for Human Cooperation and Rehabilitation, IIT, Genova, Italy \\ 3 Institute of Robotics and Mechatronics, DLR, Oberpfaffenhofen, Germany \\ 4 Technical University Munich, Department of Informatics, Garching, Germany \\ ${ }^{5}$ Cognitive Robotics Department, Delft University of Technology, Delft, The Netherlands
}

\begin{abstract}
The synergy between musculoskeletal and central nervous systems empowers humans to achieve a high level of motor performance, which is still unmatched in bio-inspired robotic systems. Literature already presents a wide range of robots that mimic the human body. However, under a control point of view, substantial advancements are still needed to fully exploit the new possibilities provided by these systems. In this paper, we test experimentally that an Iterative Learning Control algorithm can be used to reproduce functionalities of the human central nervous system - i.e. learning by repetition, after-effect on known trajectories and anticipatory behavior - while controlling a bio-mimetically actuated robotic arm.
\end{abstract}

Keywords: Motion and Motor control $\cdot$ Natural machine motion $\cdot$ Human-inspired control.

\section{Introduction}

Natural and bio-inspired robot bodies are complex systems, characterized by an unknown nonlinear dynamics and redundancy of degrees of freedom (DoFs). This poses considerable challenges for standard control techniques. For this reason, researchers started taking inspiration from the effective Central Nervous System (CNS), when designing controllers for robots $[4,5]$. In this work, we test experimentally a model-free controller intended for trajectory tracking with biomimetic robots. We prove that the required tracking performances can be matched, while presenting well-known characteristics of human motor control system, i.e. learning by repetition, mirror-image aftereffect, and anticipatory behavior. We do that by presenting experiments on a robotic arm with two degrees of freedom, each of which is actuated by means of a bio-mimetic mechanism replicating the behavior of a pair of human muscles [7] (Fig. 1(a)).

\section{From Motor Control to Motion Control}

Taking inspiration from the human CNS, we aim at designing a controller able to replicate the characteristics of paleokinetic level of Bernstein classification [2]. This provides reflex function and manages muscle tone, i.e. low level feedback and dynamic inversion. We want to do that by reproducing salient features observed in humans.

Learning by repetition [10] (behavior (i)) is the first feature we are interested into. CNS is able to invert an unknown dynamics over a trajectory, just by repeating it several 


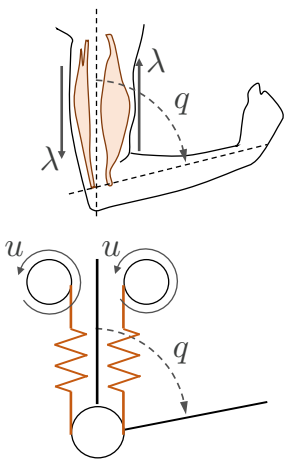

(a) Biomimetic mech.

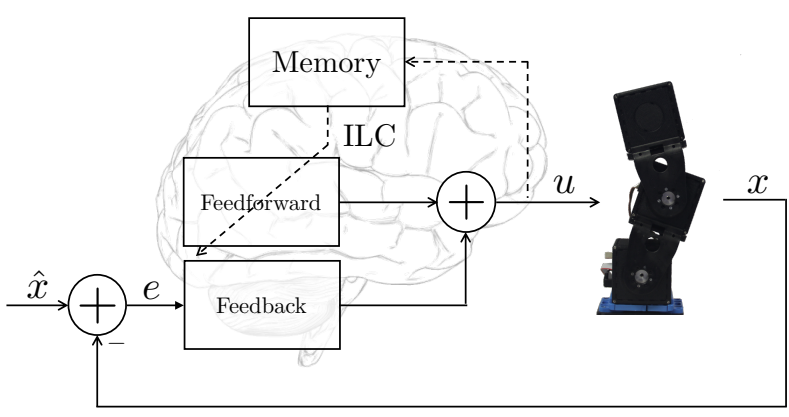

(b) Control architecture

Fig. 1. The synergy between human musculoskeletal system and the CNS can be imitated by a bio-mimic robot and a proper controller mixing anticipatory (feedforward) and reactive (feedback) actions.

times. This is clear in experiments where an unknown force field is applied to a subject's arm, and she or he is instructed to sequentially reach to track a point in space. In every repetition the tracking is improved until an almost perfect performance is recovered.

Anticipatory behavior [8] (behavior (ii)) is the second characteristic we want to reproduce. The CNS can anticipate the necessary control action relying on motor memory, rather than always reacting to sensory inputs. In control terms this means relying more on feed-forward than on feedback. In humans this characteristic tends to appear more strongly when the motor memory increases.

Finally, humans present aftereffect over a learned trajectory [9] (behavior (iii)). By removing the force field, subjects exhibit deformations of the trajectory specular to the initial deformation due to the force field introduction. This behavior is called mirrorimage aftereffect and is the third characteristic we aim at reproducing.

Fig. 1(b) shows the control architecture. We suppose no a priori knowledge of system dynamics. We just read the joint evolution and velocity $x \in \mathbb{R}^{2 n}$, and we produce a motor action $u \in \mathbb{R}^{n}$. The purpose of the controller is to perform dynamic inversion of the system, i.e. computing the control action $\hat{u}:\left[0, t_{\mathrm{f}}\right) \rightarrow \mathbb{R}^{m}$ able to track a given desired trajectory $\hat{x}:\left[0, t_{\mathrm{f}}\right) \rightarrow \mathbb{R}^{2 n}$. This has to be done by repeating several times the same task and performing it better each time (learning by repetition). To implement this feature, we propose a control law based on Iterative Learning Control (ILC) [3]: $u_{i+1}=u_{i}+\Gamma_{\mathrm{FFp}} e_{i}(t)+\Gamma_{\mathrm{FFd}} \dot{e}_{i}(t)+\Gamma_{\mathrm{FBp}} e_{i+1}(t)+\Gamma_{\mathrm{FBd}} \dot{e}_{i+1}(t)$. We call $u_{i}$ and $e_{i} \triangleq \hat{x}-x_{i}$ the control action and the error at the $i-$ th repetition of the task. $\Gamma_{\mathrm{FFp}} \in \mathbb{R}^{m \times 2 n}$ and $\Gamma_{\mathrm{FFd}} \in \mathbb{R}^{m \times 2 n}$ are the PD control gains of the iterative update while $\Gamma_{\mathrm{FBp}} \in \mathbb{R}^{m \times 2 n}$ and $\Gamma_{\mathrm{FBd}} \in \mathbb{R}^{m \times 2 n}$ are the PD feedback gains. We analyzed the theoretic control implications of using similar algorithms in $[1,6]$.

\section{Experimental Results}

The goal of the experiments is to prove that the considered ILC-based algorithm can reproduce the discussed human-like behaviors when applied to a biomimetic hardware. The algorithm is applied to a two degrees of freedom planar arm, with bio-mimetic actuation. More specifically, the mechanism mimics a pair of human muscles. The available control input $u$ has been proven to be equivalent to the corresponding signal in $\lambda$-model of human muscles [7]. We consider the following gains for the algorithm $\Gamma_{\mathrm{FFp}}$ is 


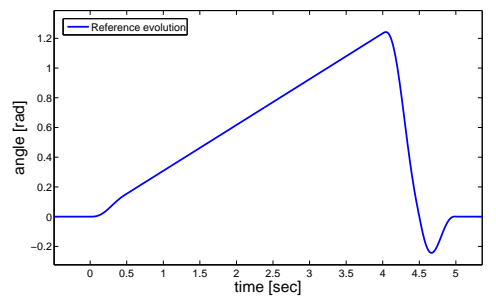

(a) Reference Trajectory

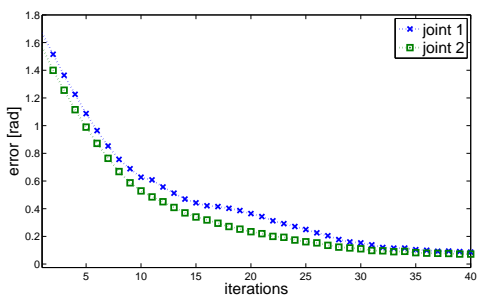

(c) Error evolution

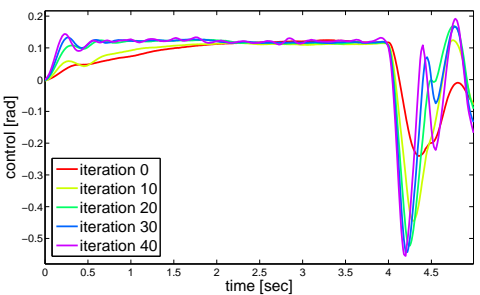

(b) Control joint 1

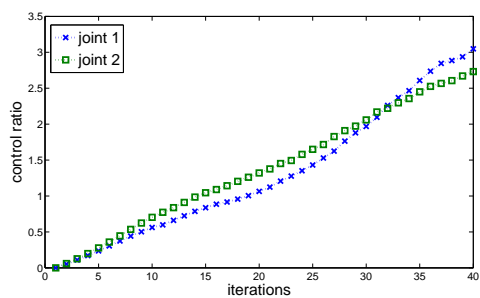

(d) Feedforward and feedback ratio

Fig. 2. Experimental results. (a) shows the reference trajectory. (b) reports the evolution of control input at joint 1. (c) shows the error over 40 iterations (behavior (i), learning by repetition). (d) depicts the ratio between reactive and anticipatory actions (behavior (ii)).

$\operatorname{blkdiag}([1,0.1],[1.25,0.0375]), \Gamma_{\mathrm{FFd}}$ is $\operatorname{blkdiag}([0.1,0.001],[0.0375,0.001]), \Gamma_{\mathrm{FBp}}$ is blkdiag $([0.25,0.025],[0.25,0.025])$, and $\Gamma_{\mathrm{FBd}}$ is blkdiag $([0.025,0.001],[0.025,0.001])$. The desired trajectory (same for both joints) is shown in Fig. 2(a). Note that this is a very challenging reference, having large amplitudes and abrupt changes in velocities. For performance evaluation we use norm 1 of the tracking error. The proposed algorithm learns the task by repeating it 40 times achieving good performance. Fig. 2(b) shows the joint 1 control evolution for some meaningful iterations (similar results apply to joint 2). Fig. 2(c) proves that the system implements learning by repetition (behavior (i)), reducing the error exponentially to 0 by repeating the same movement. Fig. 2(d) depicts the ratio between total feedforward and feedback action, over learning iterations. This shows the predominance of anticipatory action at the growth of sensory-motor memory (behavior (ii)). It is worth to be noticed that feedback it is not completely replaced by feedforward, which is coherent with many physiological evidences (e.g. [10]).

To test the presence of mirror-image aftereffect (behavior (iii)) we introduced an external force field after the above discussed learning process. This field was generated as shown by Fig. 3(a), by two springs connected in parallel to the second joint. Fig.3(b) shows the robot's end effector evolution obtained before (green) and after (red) spring introduction. The algorithm can recover the original performance after few iterations (learning process not shown for the sake of space). Finally the springs are removed, and the end-effector follows a trajectory which is the mirror w.r.t. the nominal one, of the one obtained after field introduction, therefore proving the ability of the proposed algorithm to reproduce mirror-image aftereffect (behavior (iii)).

\section{Conclusions}

In this work we proved experimentally that an ILC-based algorithm can reproduce when applied to a biobimetic hardware - several behaviors observed when the central 


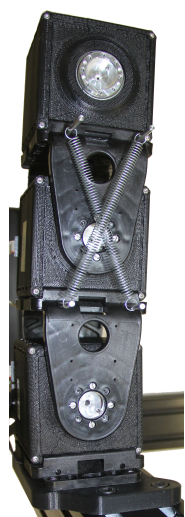

(a) Springs

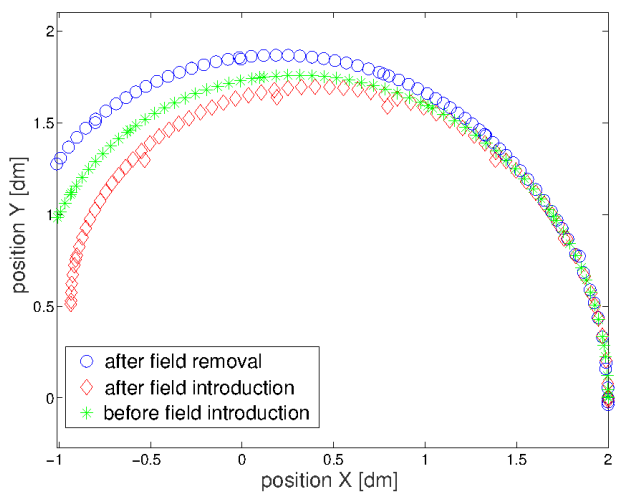

(b) Aftereffect in end effector evolutions

Fig. 3. The proposed controller presents aftereffect (behavior (iii)). Panel (a) reports the spring interconnection implementing the unknown force field, and Panel (b) end effector evolutions.

nervous system controls the muscle-skeletal system - namely learning by repetition, experience-driven shift towards anticipatory behavior, and aftereffect.

Acknowledgments. This project has been supported by European Union's Horizon 2020 research and innovation programme under grant agreement 780883 (THING) and 871237 (Sophia), by ERC Synergy Grant 810346 (Natural BionicS) and by the Italian Ministry of Education and Research (MIUR) in the framework of the CrossLab project (Departments of Excellence).

\section{References}

1. Angelini, F., Della Santina, C., Garabini, M., Bianchi, M., Gasparri, G.M., Grioli, G., Catalano, M.G., Bicchi, A.: Decentralized trajectory tracking control for soft robots interacting with the environment. IEEE Transactions on Robotics 34(4), 924-935 (2018)

2. Bernstein, N.A.: Dexterity and its development. Psychology Press (2014)

3. Bristow, D.A., Tharayil, M., Alleyne, A.G.: A survey of iterative learning control. Control Systems, IEEE 26(3), 96-114 (2006)

4. Cao, J., Liang, W., Zhu, J., Ren, Q.: Control of a muscle-like soft actuator via a bioinspired approach. Bioinspiration \& biomimetics 13(6), 066005 (2018)

5. Capolei, M.C., Angelidis, E., Falotico, E., Hautop Lund, H., Tolu, S.: A biomimetic control method increases the adaptability of a humanoid robot acting in a dynamic environment. Frontiers in neurorobotics 13, 70 (2019)

6. Della Santina, C., Bianchi, M., Grioli, G., Angelini, F., Catalano, M., Garabini, M., Bicchi, A.: Controlling soft robots: balancing feedback and feedforward elements. IEEE Robotics \& Automation Magazine 24(3), 75-83 (2017)

7. Garabini, M., Della Santina, C., Bianchi, M., Catalano, M., Grioli, G., Bicchi, A.: Soft robots that mimic the neuromusculoskeletal system. In: Converging Clinical and Engineering Research on Neurorehabilitation II, pp. 259-263. Springer (2017)

8. Hoffmann, J.: Anticipatory behavioral control. In: Anticipatory behavior in adaptive learning systems, pp. 44-65. Springer (2003)

9. Lackner, J.R., Dizio, P.: Gravitoinertial force background level affects adaptation to coriolis force perturbations of reaching movements. Journal of neurophysiology 80(2), 546-553 (1998)

10. Shadmehr, R., Smith, M.A., Krakauer, J.W.: Error correction, sensory prediction, and adaptation in motor control. Annual review of neuroscience 33, 89-108 (2010) 